eCommons@AKU

THE AGA KHAN UNIVERSITY

Faculty of Health Sciences, East Africa

School of Nursing \& Midwifery, East Africa

January 2018

\title{
Nursing and Midwifery Research Output in Africa: A Review of the Literature
}

Rose Chalo Nabirye

Makerere University

Alison Annet Kinengyere

Makerere University

Grace Edwards

Aga Khan University, grace.edwards@aku.edu

Follow this and additional works at: https://ecommons.aku.edu/eastafrica_fhs_sonam

Part of the Nursing Midwifery Commons

\section{Recommended Citation}

Nabirye, R. C., Kinengyere, A. A., Edwards, G. (2018). Nursing and Midwifery Research Output in Africa: A Review of the Literature. INTERNATIONAL JOURNAL OF CHILDBIRTH, 8(4), 236-241.

Available at: https://ecommons.aku.edu/eastafrica_fhs_sonam/257 


\title{
Nursing and Midwifery Research Output in Africa: A Review of the Literature
}

\author{
Rose Chalo Nabirye, Alison Annet Kinengyere, and Grace Edwards
}

\begin{abstract}
BACKGROUND: Nurses and midwives form the backbone of health service delivery and satisfaction with care often depends on the competencies of nurses and midwives who provide the care (World Health Organization [WHO], 2002). Healthcare has become complex, challenging, and demanding across diverse sociocultural and socioeconomic changes and environments. To optimize their impact, nurses and midwives therefore need to be prepared with evidence-based competences. Nursing research therefore, is the cornerstone for evidence-based practice and for establishing the professional status of nursing and building research capacity. However, although nursing research began in the 19th century, it is limited in Africa, with little evidence generated to inform policy and practice. Although nursing and midwifery education in the region has advanced with graduate and postgraduate level education, little is known about nursing and midwifery research conducted in universities and where to find such research.
\end{abstract}

OBJECTIVES: The literature review aimed at quantifying and identifying the types and gaps in nursing and midwives' research and publications in Africa.

METHODS: Articles published between January 1, 2007 and January 31, 2017 were retrieved from PubMed and Cumulative Index to Nursing and Allied Health Literature (CINAHL) with a search strategy that employed four sets of terms: (a) terms identifying published research done by nurses or midwives, or both; (b) terms identifying types of publications; (c) terms identifying filters for African countries; and (d) filters for publication dates. We also searched Google Scholar to capture nonindexed sources.

RESULTS AND CONCLUSIONS: There is evidence of an increasing number of African nurses and midwives publishing research; however, much of this research may not be readily available. The promotion of nursing research and capacity building/mentorship in research for nurses and midwives is recommended to increase nurses' and midwives' skills to critically evaluate research and apply the best evidence to their practice.

KEYWORDS: nursing; midwifery; nurse; midwife; nursing research; Africa

\section{BACKGROUND}

Nurses and midwives form the backbone of health service delivery. The critical role of nurses and midwives in reducing mortality, morbidity, disability, promoting healthy lifestyles, and increasing access to comprehensive healthcare was noted in 2002 and 2011 by the World Health Organization (WHO) and the World Health Assembly (WHA) (WHO, 2002, 2011). The WHA then directed countries to strengthen nursing and midwifery professions, in order to maximize their contribution to the health of populations. In the same resolution, countries were urged to strengthen nursing and midwifery professions in the areas of management/administration, education, legislation, policy and planning, regulation and practice, to meet the needs of the populations they serve (WHO, 2002, 2011).

Meeting the needs of populations, including satisfaction with care, often depends on the competencies of nurses and midwives who provide the care (WHO,

INTERNATIONAL JOURNAL OF CHILDBIRTH Volume 8 Issue 4, 2018 (C) 2018 Springer Publishing Company, LLC www.springerpub.com http://dx.doi.org/10.1891/2156-5287.8.4.236 
2002). It should be noted that healthcare in the $21 \mathrm{st}$ century has become complex, challenging, and demanding across diverse sociocultural and socioeconomic changes and environments. To optimize their impact, nurses and midwives need to be prepared with evidencebased advanced competencies in leadership, health policy, and systems, as well as in practice and education. Nursing research, the systematic search or inquiry to develop, refine, or expand knowledge about issues of importance to the nursing profession is therefore the cornerstone for evidence-based practice, thereby for quality care (Polit \& Beck, 2017).

Although research in nursing begun with Nightingale in the 19th century, limited nursing research is conducted in Africa; little evidence is generated especially in the African region to inform policy and practice for nursing and midwifery (Adejumo \& Lekalakala-Makgele, 2009). A scoping review by Sun and Larson (2015) highlighted the critical gaps in nursing and midwifery research in Africa, particularly around chronic and noncommunicable diseases. This may be linked to the lack of support and gaps in funding for nursing and midwifery research. Nursing and midwifery education in the region has advanced with the introduction of university-based bachelor- and graduate-level education where students and faculty are encouraged to conduct research. However, little is known about what types of research is conducted in universities and where to find such research.

The aim of this literature review therefore, was to document nursing and midwifery research output in Africa. Specifically, the review aimed at quantifying and identifying the type and gaps in research that has been done by African nursing students, faculty, and other members of the nursing and midwifery discipline in Africa.

\section{METHODS}

An electronic literature search strategy was conducted including the following search terms:

"nursing," "nurse," "nurses," "midwife,"

"midwifery," "midwives," research," "research output," "nursing research," "clinical nursing research," "research productivity," "dissertation," "abstract," "thesis," "theses," "publication," "poster session," "Poster presentation," "book chapter," "technical report," Africa [MESH Terms]
The search was restricted to research conducted in the last 10 years (January 1, 2007 to June 30, 2017).

After a preliminary search was conducted in PubMed, it was adapted for Cumulative Index to Nursing and Allied Health Literature (CINAHL) which allows identification of an author as a nurse after June 2009, and Google Scholar. We omitted any duplicate studies or studies that were not done in Africa. Then the papers were reviewed to identify the credentials of the authors to determine if an author was an African nurse or midwife. If the paper did not have adequate information, such as missing credentials or location of the author, institutional websites were searched to locate a person and confirm their credentials and eligibility for the study. The initial search was led by one of the authors (AK). However, all three authors were involved in the screening process. Conflicts were discussed by the all the reviewers, and consensus reached.

Those publications that met the set inclusion criteria were counted and recorded in the PRISMA flow chart (Figure 1). Qualifying studies were identified as to study type: quantitative, qualitative, or mixed methods and the geographical location of the author. Thematic analysis was used to code and stratify the papers for area of focus. Eight categories emerged and the country where the research was done was recorded.

\section{FINDINGS}

A total of 1,145 articles were retrieved from the three databases out of which only 293 articles were selected for review. Of those reviewed, 152 (52\%) had African nurses or midwives as first or second author. More than $60 \%$ of the articles with nurses and midwives as first authors were from the South Africa region, followed by West Africa (18.5\%) (See Table 1).

The types of studies were varied. Ten of the studies were systematic or integrative reviews. Of the remaining papers, 155 used quantitative methods and 128 used qualitative methods.

A thematic analysis was undertaken to explore the content of the publications. Eight categories emerged from the thematic analysis. One-third of the research (30.1\%) was on training and education, followed by workplace issues (28.3\%) and clinical practice (24.5\%). Research on infectious diseases contributed only $6.4 \%$ and there were no studies on noncommunicable diseases. 
New Prisma Chart

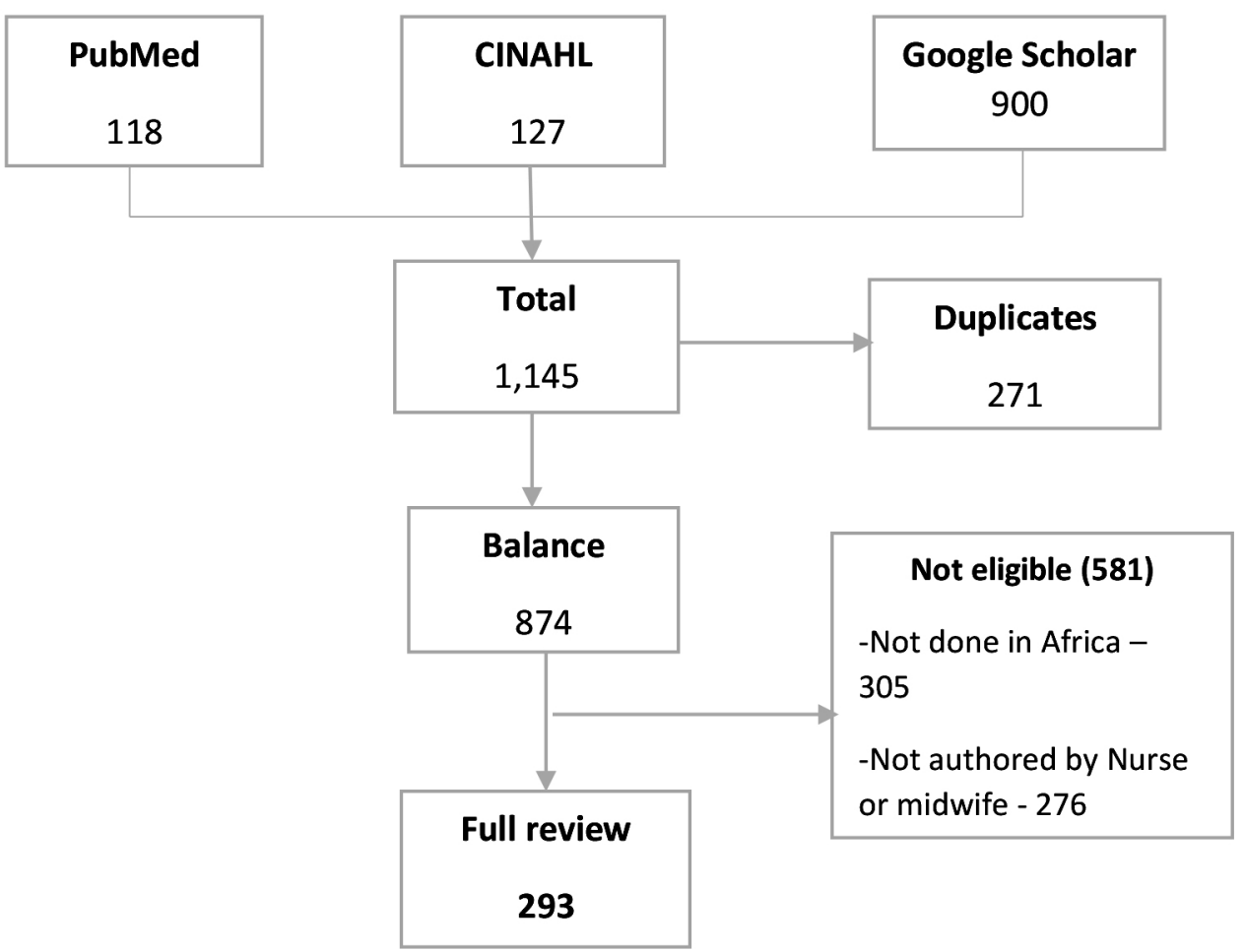

FIGURE 1. PRISMA flow chart.

TABLE 1. Quantity of Publications by African Nurse/Midwife Author and African Region

\begin{tabular}{|c|c|c|}
\hline ITEM & NUMBER & PERCENTAGE \\
\hline Nurse or midwife first author from Africa & 119 & 40.6 \\
\hline Nurse or midwife first author not from Africa & 141 & 48.1 \\
\hline $\begin{array}{l}\text { Nurse or midwife second or subsequent author } \\
\text { from Africa }\end{array}$ & 33 & 11.3 \\
\hline Total articles & 293 & 100 \\
\hline \multicolumn{3}{|c|}{ Nurse or midwife first authorship by region $(N=119)$} \\
\hline East Africa & 17 & 14.2 \\
\hline North Africa & 07 & 5.9 \\
\hline South Africa & 73 & 61.4 \\
\hline West Africa & 22 & 18.5 \\
\hline
\end{tabular}

\section{DISCUSSION}

This literature review of research published in Africa covered a 10-year period, from 2007 to 2017.

It is reassuring to see the increase in the number of nurses and midwives who are leading on research in Africa as supported by the historical review by Klopper and Gasanganwa (2015), but disappointing to note the paucity of African nurses and midwives' authors.

The fact that the large majority of African nurse or midwife authors came from South Africa and the fewest from North Africa is similar to the findings of agree with Adejumo and Lekalakala-Makgele (2009), who reported a higher number of publications from the southern region (61\%) as compared to $5.2 \%$ and $3.3 \%$ only from Western and Eastern Africa, respectively. The high percentage of publications in Southern Africa may be attributed to the longer history of nursing and midwifery education in the region as compared to other regions. Nurses and midwives educated at higher education levels are more likely to conduct research as part of their education, and therefore, they are more likely 
to publish than those trained at lower levels (Squires, Estabrooks, Gustavsson, \& Wallin, 2011).

The methodological approach of the selected papers was explored. Descriptive surveys were utilized in almost all of the quantitative papers, with the commonest approach concentrating on opinions and views of other healthcare professionals, usually other nurses and midwives. Qualitative research accounted for $43.7 \%$ ( $n=128)$ of the papers reviewed. The qualitative papers utilized validated methodological models such as ethnography and grounded theory. There was also a number of papers that were narratives, stories, or case studies $(3 \%, n=9)$. Six papers (2\%) used mixed methods. It is important to note that nurses and midwives employ both quantitative and qualitative methods in their research. Literature has demonstrated that evidence from both methods is important in nursing practice. According to Miller (2010), much as qualitative research findings cannot be generalized to populations using universal laws for populations, they can be generalized in nursing practice when adapted to care of individuals in same situations as those studied.

Surprisingly, there were only 19 papers that concentrated on the major communicable diseases prevalent in Africa: HIV, Ebola, and tuberculosis (TB). These three diseases along with tropical diseases are the major threats to health in Africa (WHO, 2016). There were no papers specifically targeting malaria, in spite of the large amount of funding that communicable disease research attracts. Olesen and Parker (2012) noted almost all health and research funding in Africa is directed toward HIV, malaria, and TB programs although these diseases account for less than $25 \%$ of mortality and morbidity figures.

The fact the studies used a combination of quantitative and qualitative approaches is encouraging that African nurses and midwives are being prepared in both research methodologies important to nursing practice. The large percent of qualitative studies may be an effective method of truly understanding the employment and care issues African nurses and midwives are dealing with. According to Miller (2010), although qualitative research findings cannot assume universal laws for populations limiting its generalizability to populations, they can be generalized in nursing practice when adapted to care of individuals in similar situations as those studied.

The paucity of actual analytic quantitative studies done by African nurses and midwives is of concern and may highlight several issues that have been identified in the literature. Very few of the nursing and midwifery papers reviewed had been funded by external bodies.
The problems associated with African nurse and midwife researchers attracting funding for research is not new and is evident in other areas of health research. Work by Kokwaro and Kariuki (2001) highlighted the deficiencies around infrastructure, research career development, and financial and education support for medical and allied health researchers. These issues are still evident today. There is also a marked disparity in the distribution of funding for research with large inequities around funding, which not based on need and variation in the country of origin for African research (Head et al., 2017). A review of research output from Africa highlighted that a large proportion of research (40\%) from Sub-Saharan Africa was generated by South Africa researchers. Nigerian publications accounted for 16\%; Kenya, 7\%; Zimbabwe, 3\%; and Ethiopia, 3\%. All other countries in Sub-Saharan African produced just over $2 \%$ of all publications (Hofmann, Kanyengo, Rapp, \& Kotzin, 2009). It seems that little has changed over the past 20 years as Horton (2000) and King (2004) pointed out that this low level of publications from Sub-Saharan Africa has been consistent in other reviews, stating that 31 of the world's 193 countries produce $97.5 \%$ of the world's most cited papers and only South Africa at number 29 represents input from Africa.

Work by Spies and colleagues stressed the importance of empowering nurses and midwives to identify and conduct nursing research to inform policy makers (Spies, Gray, Opollo, \& Mbalinda, 2015). As frontline practitioners, nurses and midwives are able to identify the issues that may directly impact the health of the population, but they need the skills to be able to critically evaluate the issues and lead research that will identify solutions to challenges. This is important for several reasons. Nurses and midwives form the largest group of health professionals worldwide, yet they are often invisible in the higher-level decision-making around policies and directives, which affects their work. Some of the factors reported to limit involvement of nurses in research include rigid power hierarchies among different health professional disciplines, lack of opportunities for graduate and postgraduate training, lack of mentors and resources (Edwards, Webber, Mill, Kahwa, \& Roelofs, 2009). However, recent evidence suggests there are examples of the growing positive influence of nursing research and scientific application in several African countries around infectious diseases which may be used as exemplars (Baltzell, McLemore, Shattell, \& Rankin, 2017).

Universities and colleges have a role to play in preparing nursing and midwifery students with the 
skills and confidence to plan, perform, and lead clinical research teams. This would require the support of clinical and academic nurse and midwifery leaders to support and empower novice researchers. There are successful initiatives that are committed to developing nursing and midwifery research expertise. One of such initiatives is the Lugina African Midwifery Research Network (LAMRN) which has developed a supportive research partnership between the United Kingdom and six African countries with the aim of supporting midwifery research, information sharing, and networking and training activities. The priorities of LAMRYN (http://lamrn.org) are

1. To support senior midwifery partners to develop research leadership skills

2. To work with midwives in target countries to identify country-level maternal health research priorities

3. To provide training for midwives to improve evidence-based practice skills

4. To develop a mentorship system for midwives to develop research projects in priority

During their work on increasing human resource capacity in African countries, Sure et al. (2016) established a network of research experts in the region, peer mentorship was initiated, and research priorities and regional action plans were identified.

Such initiatives are necessary to develop all areas of nursing and midwifery, although they are dependent on funding, which may be a challenge. In this review, $44 \%$ of the publications utilized qualitative methodology. This finding was similar to a study by Sun and Larson (2015) where $57 \%$ of the research was reported to be qualitative. Miller (2010) observed there is a misconception that findings from qualitative research lack generalizability, are not strong enough, and cannot stand alone. She asserts that although qualitative research findings do not provide laws and relationships from population representative samples, they can be applied in nursing care or practice when applied to other individuals going through the same experiences.

Nursing and midwifery involve care of individuals of all ages, families, and communities in all settings in collaboration with other healthcare professionals. It can be argued therefore, that qualitative methodology, which according to Polit and Beck (2017) is an inquiry based on experiences, realities, and viewpoints of those being studied is the most appropriate for nursing and midwifery.

\section{CONCLUSIONS AND RECOMMENDATIONS}

Although there is evidence of an increase of research by African nurses and midwives, the profile of such research is still low. The number of African nurses and midwives publishing research is growing, but much of this research may not be readily available. African nursing and midwifery research is often published in nonindexed journals especially around topics such as chronic illnesses and children's conditions (Sun et al., 2016). Many nurses and midwives may lack the skills in being able to access this literature and so may miss valuable information that may enhance their nursing care.

The role of nurses and midwives is crucial to improving patient care and outcomes. There is need to work toward ensuring that every African nurse and midwife has the skills to critically evaluate research and apply the best evidence to their practice and develop and support those nurses and midwives who are committed to research. The promotion of nursing research and capacity building/mentorship in research for nurses and midwives are recommended.

\section{REFERENCES}

Adejumo, O., \& Lekalakala-Makgele, E. (2009). A 2-decade appraisal of African Nursing Scholarship: 19862006. Journal of Nursing Scholarship, 41(1), 64-69. doi:10.1111/j.1547-5069.2009.01252.x

Baltzell, K., McLemore, M., Shattell, M., \& Rankin, S. (2017). Impacts on global health from nursing research. The American Journal of Tropical Medicine and Hygiene, 96(4), 765-766. doi:10.4269/ajtmh.16-0918

Edwards, N., Webber, J., Mill, J., Kahwa, E., \& Roelofs, S. (2009). Building capacity for nurse-led research. International Nursing Review, 56, 88-94. doi:10.1111/j.14667657.2008.00683.x

Head, M. G., Goss, S., Gelister, Y., Alegana, V., Brown, R. J., Clarke, S. C., . . . Tatem, A. J. (2017, August). Global funding trends for malaria research in sub-Saharan Africa: A systematic analysis. Lancet Global Health, 5(8), e772-e781. doi:10.1016/S2214-109X(17)30245-0

Hofman, K. J., Kanyengo, C. W., Rapp, B. A., \& Kotzin, S. (2009). Mapping the health research landscape in SubSaharan Africa: A study of trends in biomedical publications. Journal of the Medical Library Association, 97(1), 41-44. doi:10.3163/1536-5050.97.1.007

Horton, R. (2000, September). North and south: Bridging the information gap. Lancet, 355(9222), 2231-2236. doi:10.1016/S0140-6736(00)02414-4 
King, D. A. (2004, July). The scientific impact of nations: What different countries get for their research spending. Nature, 430(6997), 311-316. doi:10.1038/430311a

Klopper, H., \& Gasanganwa, M. (2015). State of the world in nursing research. Rwanda Journal Series F: Medicine and Health Sciences, 2(2). doi: 10.4314/rj.v2i2.2F

Kokwaro, G., \& Kariuki, S. (2001). Medical research in Africa: Problems and some solutions. Malawi Medical Journal: The Journal of Medical Association of Malawi, 13(3), 40.

Miller, W. R. (2010). Qualitative research findings as evidence: Utility in nursing practice. Clinical Nurse Specialist, 24(4), 191-193. doi:10.1097/NUR.0b013e3181e36087

Olesen, O. F., \& Parker, M. I. (2012). Health research in Africa: Getting the priorities right. Tropical Medicine and International Health, 17(9), 1048-1052. doi:10.1111/j.13653156.2012.03027.x

Polit, D. F., \& Beck, C. T. (2017). Nursing research: Generating and assessing evidence for nursing practice. Philadelphia, PA: LWW.

Spies, L. A., Gray, J., Opollo, J., \& Mbalinda, S. (2015). Uganda nursing research agenda: A Delphi study. International Nursing Review, 62(2), 180-186. doi:10.1111/inr.12167

Squires, J. E., Estabrooks, C. A., Gustavsson, P., \& Wallin, L. (2011). Individual determinants of research utilization by nurses: A systematic review update. Implementation Science, 6(1), 1. doi:10.1186/1748-5908-6-1

Sun, C., Dohm, J., Omani, G., Malata, A., Klopper, H., \& Larson, E. (2016, March). Clinical nursing and midwifery research: Grey literature in African countries. International Nursing Review, 63(1), 104-111. doi:10.1111/inr.12231

Sun, C., \& Larson, E. (2015). Clinical nursing and midwifery research in African countries: A scoping review. International Journal of Nursing Studies, 52(5), 1011-1016. doi:10.1016/j.ijnurstu.2015.01.012
World Health Organization. (2002). Resolution WHA54.12. Strengthening nursing and midwifery. Fifty-fourth World Health Assembly, Geneva. Retrieved from http://www. who.int/hrh/resources/WHA54-12.pdf

World Health Organization. (2011). Resolution WHA 64.7. Strengthening nursing and midwifery. Sixty-fourth World Health Assembly, Geneva. Retrieved from http:// apps.who.int/gb/ebwha/pdf_files/WHA64/A64_R7-en. pdf?ua $=1$

World Health Organization. (2016). WHO African region communicable diseases cluster annual report 2016. Retrieved from https://afro.who.int/publications/whoafrican-region-communicable-diseases-cluster-annualreport-2016

Disclosure. There are no conflicts of interest to disclose and all authors contributed to writing this manuscript.

Correspondence regarding this article should be directed to Grace Edwards, RN, RM, ADM, Cert Ed, MEd, PhD, Professor of Midwifery Education and Practice, Aga Khan University, Uganda Campus, Colonel Muammar Gadafi Road, Kampala 8842, Uganda. E-mail: grace.edwards@ aku.edu

Rose Chalo Nabirye, RNM, ADHSM, BScN, MPH, PhD, Senior Lecturer, Department of Nursing, School of Health Sciences, Makerere University, Kampala, Uganda.

Alison Kinengyere, PhD, Librarian, Sir Albert Cook Library, College of Health Sciences, Makerere University, Kampala, Uganda.

Grace Edwards, RN, RM, ADM, Cert Ed, M Ed, PhD, Professor of Midwifery Education and Practice, Aga Khan University, Kampala, Uganda. 